\title{
Bienestar psicológico en las personas mayores no dependientes y su relación con la autoestima y la autoeficacia
}

\author{
Psychological well-being in non-dependent active elderly \\ individuals and its relationship with self-esteem and self-efficacy
}

María Mesa-Fernández ${ }^{1}$

Javier Pérez-Padilla ${ }^{2}$

Cristina Nunes ${ }^{3}$

Susana Menéndez ${ }^{4}$
${ }^{1}$ Departamento de Psicologia e Ciências da Educação, Universidade do Algarve. Estr. da Penha 139. 8005-139 Faro Algarve Portugal.

maria.mesa.fdz@gmail.com ${ }^{2}$ Departamento de

Psicología, Universidad de Jaén. Jaén Andaluzia Espanha.

${ }^{3}$ Universidade do Algarve. Faro Algarve Portugal.

${ }^{4}$ Departamento de

Psicología Evolutiva y de la

Educación, Universidad de

Huelva. Huelva Espanha.

\begin{abstract}
The aim of this study was to examine the relationship between psychological well-being, self-efficacy and self-esteem in non-dependent individuals over the age of 60 . The participants included 148 seniors between 60 and 96 years of age. Data were collected through the following questionnaires: autonomy and physical and social activity scale, self-efficacy for aging scale, self-esteem scale, and the Spanish version of the wellness psychology scale. The results suggest that psychological well-being was not associated with age, but with a set of psychological factors. Psychological well-being was associated with health perception, physical and sports activities, self-efficacy, self-esteem and autonomy. The last three variables are predictors of psychological well-being. Consequently, self-efficacy and self-esteem are considered promoters of physical, psychological and social well-being, encouraging participation in physical and sports, recreational, social and cognitive activities promoting wellness and, ultimately, active aging.
\end{abstract}

Key words Psychological well-being, Active aging, Elderly individuals, Self-esteem, Self-efficacy for aging
Resumen Este estudio tuvo como objetivo examinar las relaciones entre el bienestar psicológico, la autoeficacia para envejecer y la autoestima en personas mayores de 60 años no dependientes. Han participado 148 personas con edades comprendidas entre 60 y 96 años, completando una batería de instrumentos relacionados con la autonomía, el grado y tipo de actividad, la autoeficacia para afrontar el proceso de envejecimiento, la autoestima y el bienestar psicológico. Los resultados indican que el bienestar psicológico no está asociado con la edad, sino con un conjunto de factores de corte psicológico. En concreto la percepción de la salud, las actividades físico-deportivas, la autoeficacia para envejecer, la autoestima y la autonomía se relacionan con el bienestar psicológico, aunque sólo los tres últimos indicadores lo explican. Por tanto la autoeficacia y la autoestima funcionan como promotores del bienestar físico, psicológico y social, de manera que resulta fundamental motivar la participación de los mayores en actividades físico-deportivas, recreativas, sociales y cognitivas promoviendo así su bienestar.

Palabras clave Bienestar psicológico, Envejecimiento activo, Personas mayores, Autoestima, Autoeficacia para envejecer 


\section{Introducción}

Desde la mitad del siglo XX, el envejecimiento demográfico ha recibido una considerable atención debido a los desafíos sociales que representa ${ }^{1}$. Se estima que, en el 2050, la población mundial de personas mayores de 60 años se triplicará, pasando de los 60 millones a los 200 millones, y la población mayor de 80 años será de 400 millones, pasando del $11 \%$ al $22 \%{ }^{2}$.

En el caso de Europa, la población mayor de 65 años se incrementará del actual $18 \%$ a un $28 \%$, y los mayores de 80 años pasaran del 5\% al 12\% ${ }^{3}$. En España se estima que existan 8,5 millones de personas mayores de 65 años $(18,2 \%)$ y las proyecciones demográficas del Instituto Nacional de Estadística ${ }^{4}$, indican que en el año 2065 este colectivo va a ser un $38,70 \%$ de la población total. Sin embargo, este aumento no supone que envejecer sea sinónimo de ser dependiente. El 73,90\% de la población española mayor de 65 años no padece enfermedades graves o demencias, ni necesita apoyo o ayuda de otras personas para las actividades de la vida diaria (AVD), como comer, vestirse, asearse o levantarse ${ }^{5}$.

El proceso de envejecimiento es un fenómeno multidimensional y heterogéneo. La experiencia vital y los diversos factores socioculturales, genéticos y biológicos determinan el proceso de envejecimiento y la calidad de vida en la vejez ${ }^{1,6-8}$. La participación activa en los ámbitos social, económico, político y cultural, entre otros, está asociada con una buena salud, un buen funcionamiento cognitivo, calidad y satisfacción con la vida y bienestar psicológico. Además, favorece que las personas mayores experimenten sentimientos y actitudes positivas, optimizando los mecanismos de autorregulación y autocontrol y mejorando el afrontamiento a los problemas y la capacidad de adaptación en esta etapa vital ${ }^{1}$.

En consecuencia, es importante el análisis del bienestar en la vejez entendido como un constructo que incluye un aspecto psicológico que influye en la optimización y desarrollo de las personas mayores. Carol Ryff 9 se refiere al bienestar psicológico (BP) como el esfuerzo de perfeccionar el propio potencial, relacionado con el propósito de vida, donde la vida de la persona adquiere un significado para sí misma, mediante ciertos esfuerzos de superación y consecución de metas valiosas. El BP es conceptualizado a través de una visión eudaimónica del bienestar y explicado por un modelo multidimensional de desarrollo personal donde se especifican seis dimensiones psicológicas propias de la persona: autoaceptación (evaluaciones positivas de sí mismo y de la vida pasada; conocer y aceptar los diferentes aspectos de la persona), autonomía (autodeterminación, independencia y autorregulación, capacidad de pensar y actuar de forma autónoma, resistiendo a las presiones sociales), dominio del ambiente (habilidad para crear ambientes favorables donde poder satisfacer las necesidades y valores, aprovechando las oportunidades, sensación de control sobre los acontecimientos e influencias sobre el contexto), relaciones positivas (posibilidad de generar vínculos confiables, cálidos y satisfactorios con otras personas), propósito de vida (poseer metas y un sentido direccional e intencional, siendo capaz de dar sentido a sus experiencias presentes y pasadas y comprender el propósito de su vida) y crecimiento personal (sentido de crecimiento y desarrollo continuo del propio potencial, y usar las diferentes capacidades al enfrentarse a nuevos desafíos y tareas característica de cada etapa evolutiva) ${ }^{9}$.

La percepción del BP está influida por indicadores sociodemográficos, como la edad, el sexo, el estado civil y el nivel educativo. Diversos estudios han revelado que las dimensiones de propósito de vida y crecimiento personal muestran variaciones relacionadas con la edad, mientras que la autonomía y el dominio del ambiente permanecen relativamente estables ${ }^{9,10-12}$. Durante el proceso de envejecimiento la persona va realizando reajustes de las propias metas personales mediante la compensación de las pérdidas, asimismo, el contexto ofrece oportunidades limitadas, lo cual no favorece las experiencias significativas, pudiendo provocar efectos negativos en el crecimiento personal, y en menor medida, en el propósito de vida ${ }^{13,14}$. De acuerdo con diversos autores ${ }^{9,14}$, los hombres tienden a presentar valores más elevados en autonomía, crecimiento personal y autoaceptación, mientras que las mujeres destacan por el bienestar en las relaciones sociales. Por otro lado, las personas casadas tienden a percibirse de manera positiva en el dominio del ambiente, autoaceptación, propósito de vida y crecimiento personal ${ }^{15,16}$. Además, las personas con mayor nivel educativo presentan una mayor salud percibida ${ }^{17}$, un BP satisfactorio basándose en la autoaceptación, propósito de vida y crecimiento personal ${ }^{11,12,16}$, debido a que poseen más recursos internos y externos para lidiar con las situaciones problemáticas a través de las estrategias adecuadas de afrontamiento y autorregulación.

Por otro lado, cuando las personas presentan niveles elevados de autoestima tienden a experimentar un mayor bienestar, y una buena salud en general ${ }^{18-20}$. Percibir y poseer una buena salud tiende a promover niveles elevados de autoefica- 
cia, permitiendo que la persona participe activamente en actividades físicas, sociales y recreativas, provocando un aumento de bienestar ${ }^{21,22}$. En este sentido, la autoeficacia, en parte, está relacionada con la autoestima donde el nivel de autoeficacia determina el grado de implicación y persistencia en una tarea, afectando a la resolución de la misma y teniendo una repercusión en la autoestima ${ }^{8}$, y a su vez de nuevo, en el bienestar. De este modo, una elevada autoeficacia posibilita una buena percepción de las propias capacidades, con pensamientos motivadores para la acción, sin embargo, una baja autoeficacia puede promover sentimientos negativos para superar las dificultades relacionándose negativamente con la autoestima ${ }^{8}$.

En esta misma línea, la autoeficacia tiene un papel protector en las conductas perjudiciales para la salud (i.e. tabaquismo, sedentarismo, dieta inadecuada, consumo de alcohol), en la regulación emocional ${ }^{23-25}$, y en la promoción de la actividad física ${ }^{26}$. Respecto a esta última dimensión, Queiroz et al. ${ }^{27}$ revelan que una mayor edad se encuentra relacionada con una menor actividad física, explicándose esta relación por los cambios físicos y funcionales del envejecimiento. Otras investigaciones realizadas demuestran que una actividad física ligera como caminatas, alargamientos y tonificación muscular producen efectos positivos en el bienestar ${ }^{28,29}$. En este sentido, la actividad física es una dimensión fundamental a la hora de entender el bienestar de la persona mayor, debido a que existe una relación positiva con esta dimensión. Asimismo, la actividad física modula los niveles de autoestima y mejora las capacidades de aprendizaje, el contacto social, el autoconcepto, el equilibrio emocional y afectivo, reduciendo de esta manera los síntomas de ansiedad y depresión ${ }^{28,30,31}$.

En relación a la participación social, las investigaciones revelan que la colaboración en actividades comunitarias, como los programas educativos, de salud y artístico-culturales, mejoran el nivel de salud reduciendo el nivel de dependencia, a través de la prevención de accidentes, la promoción de la salud y la actividad física. Además, estos programas favorecen el contacto social creando redes de apoyo social, reduciendo la soledad y la depresión, promoviendo el bienes$\operatorname{tar}^{31-34}$. Otros estudios sugieren que las personas que participan en los programas de las Universidades Seniors, además de actualizar y adquirir nuevos conocimientos y competencias, crean y favorecen el establecimiento de redes sociales, atenuando así la soledad, y estimulando el sentimiento de actividad, la percepción de autoefi- cacia, la autoestima y el bienestar ${ }^{35-37}$. Concretamente, Clemente et al. ${ }^{38}$ indican que las personas que sienten mayor satisfacción con la calidad de su red de apoyo social, presentan mejores niveles de salud percibida y de bienestar.

En suma, los estudios con personas mayores no dependientes muestran que el envejecimiento no es una etapa exclusiva de pérdidas, sino de adaptación, oportunidades y crecimiento, donde la participación activa en los diversos contextos de la sociedad, favorece su calidad de vida y su bienestar. Para ello, a través de las directrices de la OMS, existen programas de promoción del envejecimiento activo, con el objetivo de fomentar la salud, la participación social, la actividad física y cognitiva, entre otros, y así apoyar una autoimagen y autoestima positiva y, en conclusión, el bienestar. En este sentido, los objetivos de este estudio son:

- Caracterizar y analizar el perfil sociodemográfico de una muestra de personas mayores no dependientes.

- Analizar las relaciones entre el bienestar psicológico y dimensiones sociodemográficas, de salud (autonomía, actividad y salud percibida) y psicológicas (autoestima y autoeficacia).

- Explorar los posibles factores que explican el grado de bienestar psicológico.

\section{Método}

\section{Participantes}

Han participado 148 personas, mayores de 60 años no dependientes, residentes en la provincia de Huelva. Los participantes tenían edades comprendidas entre los 60 y 96 años $(M=70,34$ años; $D T=7,41)$ y el $51 \%$ eran mujeres.

\section{Instrumentos}

Cuestionario breve por items de la Batería Mínima de Evaluación en la Vejez (BEV) ${ }^{39}$. Compuesto por siete ítems de la BEV que recoge la información sobre el nivel de autonomía (1 ítem), salud percibida (3 ítems), actividad física (1 ítem), cuidado informal (1 ítem) y de participación (1 ítem); para este trabajo se han seleccionado los primeros cinco ítems de acuerdo con los objetivos del estudio. El ítem de autonomía está compuesto dos cuestiones dicotómicas sobre la necesidad (sí/no) y tipo de ayuda (formal/informal) y una cuestión cerrada acerca de la frecuencia de ayuda (diariamente y conviven/diariamente, pero no conviven/varias veces por semana). En cuanto a la 
salud percibida, el ítem sobre la salud en general formulado en una escala Likert de cuarto opciones de respuesta, 1 (Muy buena) a 4 (Muy mala), el resto de los ítems relativamente a la comparación de la salud, se presentan en una escala Likert de cinco puntos 1 (Mucho mejor) a 5 (Mucho peor). La actividad física presentaba un ítem permitiendo recabar información sobre el tipo de ejercicio físico, regularidad y frecuencia, medido en horas por semana. Respecto a las actividades de cuidado informales, este ítem proporciona el nivel de responsabilidad y frecuencia en las tareas de cuidado de otras personas como pareja, nietos y otras personas mayores. Por último, en relación a la participación en actividades comunitarias, recreativas y/o cognitivas, estaba formado por un ítem dicotómico (sí/no) sobre la participación en general y una pregunta abierta sobre las actividades en las cuales participa la persona.

Escala de Autoeficacia para Envejecer ${ }^{39}$. La escala permite evaluar el control percibido sobre el proceso de envejecimiento. Compuesta por diez ítems $(\alpha=, 91)$, presentados en una escala Likert de cuatro opciones de respuesta, desde 1 (Nada) a 4 (Mucho), y distribuidos en cuatro dimensiones: salud (2 ítems; ,63), cognitiva ( 2 ítems; $\alpha$ $=, 62$ ), físico/funcional (3 ítems; $\alpha=, 81$ ) y socio-emocional ( 3 ítems; $\alpha=, 69$ ). Presenta una puntuación final que varía de 10-40.

Escala de Autoestima de Rosenberg ${ }^{40}$. Compuesta por diez ítems $(\alpha=, 74)$ presentados en una escala Likert de cuatro opciones de respuesta, desde 1 (Muy de acuerdo) a 4 (Totalmente en desacuerdo). Presenta una puntuación final mínima de 10 y máxima de 40.

Escala de Bienestar Psicológico (Psychological Well-Being Scales) - Versión Española breve ${ }^{41}$. Escala compuesta por 29 ítems $(\alpha=, 86)$, presentados por una escala Likert de seis opciones de respuesta, desde 1 (Totalmente en desacuerdo) a 6 (Totalmente de acuerdo). Contempla seis dimensiones: autoaceptación ( 4 ítems; $\alpha=, 80$ ), relaciones positivas ( 5 ítems; $\alpha=0.26$ ), autonomía ( 6 ítems; $\alpha=0.32$ ), dominio del ambiente ( 5 ítems; $\alpha=, 48$ ), crecimiento personal (4 ítems; $\alpha=, 53$ ) y propósito de vida (5 ítems; $\alpha=, 82$ ).

\section{Procedimiento}

La muestra fue seleccionada por conveniencia atendiendo a los criterios de inclusión de ser mayor de 60 años y no dependiente. La muestra fue seleccionada con ayuda de instituciones locales que realizan actividades para personas mayores (ej. asociaciones de vecinos, asociaciones de mayores, Cruz Roja y Aula de la Experiencia de la
Universidad de Huelva). Cada profesional seleccionó un grupo de usuarios de acuerdo a los criterios de inclusión del estudio, y después de recabar su colaboración voluntaria, se concertó una cita en las dependencias del centro a la que acudió un miembro del equipo de investigación. Tomando en consideración las características de la persona mayor, la administración de los cuestionarios pudo ser en modo entrevista (con una duración de 45 minutos) o autoadministrado (con una duración de 15 minutos). La batería en el Aula de la Experiencia de la Universidad de Huelva, fue de modo auto-administrada estando presente en todo momento algún miembro del equipo de investigación. Antes de administrar la batería de instrumentos, los participantes fueron informados sobre los objetivos de la investigación, confidencialidad y anonimato de sus respuestas.

\section{Plan de análisis}

Todos los análisis fueron realizados con el paquete estadístico IBM SPSS para Windows (IBM SPSS 20.0). Los resultados descriptivos referidos a las dimensiones objeto de estudio se realizaron de acuerdo al tipo de variable, cualitativo o cuantitativo, ofreciéndose porcentajes, medias, desviaciones típicas y mínimos y máximos. Para realizar las comparaciones de medias se utilizaron las pruebas paramétricas y no paramétricas. Para analizar las relaciones entre variables cuantitativas y cualitativas, se utilizaron el coeficiente $r$ de Pearson y $r$ de Spearman, respectivamente. Por último, se ha realizado una regresión múltiple jerárquica, para analizar y determinar las variables que explican el bienestar psicológico.

\section{Resultados}

\section{Perfil sociodemográfico de los participantes}

La mayoría de los participantes en el estudio estaban casados $(71,0 \%)$, siendo la media en años de relación matrimonial de 41,02 años (DT $=13,02)$. Los participantes residían en su propia casa con su pareja $(53,52 \%)$, tenían dos hijos $(D T=1,13$; amplitud = 1-6) y dos nietos $(D T$ $=2,44$; amplitud $=0-10)$. El nivel educativo era medio-bajo, la mayoría había completado la enseñanza secundaria obligatoria $(28,76 \%)$ o bien tenían estudios primarios (26,02\%).

Con respecto a las AVD, el $61,45 \%$ de los participantes no necesitaba ni recibía apoyo de otra persona. Los que necesitaban ayuda $(36,48 \%)$, se apoyaban en una ayuda formal $(73,91 \%)$ con una 
frecuencia semanal $(56,66 \%)$, es decir, tenían a una persona contratada para limpiar la casa, cocinar o cuidar de otros familiares. En cuanto al nivel de actividad física, la mayoría realizaba una actividad física ligera (50\%) con una frecuencia superior a tres horas por semana $(37,83 \%)$. Los participantes percibían su estado de salud como bueno $(60,81 \%)$, siendo igual que el año pasado $(65,54 \%)$ y señalando que se encontraban mejor de salud en comparación con el resto de iguales $(53,37 \%)$. Los análisis descriptivos de las puntaciones obtenidas en los cuestionarios utilizados en la presente investigación se presentan en la Tabla 1.

\section{Relaciones entre el Bienestar Psicológico y dimensiones sociodemográficas, de salud y psicológicas}

El bienestar psicológico se relacionó positivamente entre todas las subescalas y con las variables de estudio, autoestima, autoeficacia, actividad física y salud percibida (Tabla 2). A ex-

Tabla 1. Descriptivos de las escalas utilizadas.

\begin{tabular}{lrrrc}
\hline & M & DP & Mín. & Máx. \\
\hline Autoeficacia & 26,36 & 5,70 & 10 & 38 \\
Autoestima & 31,34 & 3,76 & 18 & 40 \\
BP Autoaceptación & 19,30 & 3,35 & 9 & 24 \\
BP Relaciones positivas & 19,76 & 3,66 & 6 & 28 \\
BP Autonomía & 25,57 & 4,66 & 11 & 36 \\
BP Dominio del ambiente & 22,16 & 3,9 & 8 & 30 \\
BP Crecimiento personal & 18,67 & 3,20 & 8 & 24 \\
BP Propósito de vida & 18,80 & 3,72 & 6 & 24 \\
\hline
\end{tabular}

cepción de las subescalas, crecimiento personal, relaciones positivas, y autonomía, las cuales no presentaron asociaciones para la autoeficacia, actividad física y salud percibida, respectivamente.

Asimismo, la autoestima se relacionó positivamente con a la salud percibida, y las variables actividad física, salud percibida, autoeficacia, se relacionaron positivamente entre ellas. Por otro lado, la edad se correlacionó negativamente con la subescala de propósito de vida, actividad física, salud percibida y autoeficacia.

Tal como se puede observar en la Tabla 3, aquellos participantes con una edad igual o superior a los 75 años percibieron un menor nivel de salud, una menor actividad física y una menor autoeficacia en comparación con los sujetos del grupo 60-74 años. Con respecto al nivel de salud percibida de las personas mayores con un alto nivel de estudios, fue superior en comparación con aquellos que únicamente habían cursado la enseñanza primaria. Tanto la salud percibida como la autoestima, la autoeficacia y el dominio del ambiente de las personas mayores que no recibían ayuda para las AVD obtuvieron puntuaciones elevadas en comparación con aquellas que sí la percibían. Por otro lado, las puntuaciones del nivel de salud percibida, la autoestima y la autoeficacia se distribuyeron uniformemente de acuerdo con el sexo y el estado civil de los participantes, por lo que no se encontraron diferencias relevantes.

\section{Factores que explican el grado de Bienestar Psicológico}

En la Tabla 4 se presenta el análisis de regresión lineal jerárquica siendo las diferentes subescalas del bienestar psicológico las varia-

Tabla 2. Correlaciones de Pearson entre el bienestar psicológico y las variables estudiadas.

\begin{tabular}{|c|c|c|c|c|c|c|c|c|c|c|c|c|}
\hline \multicolumn{13}{|c|}{ Bienestar Psicológico (BP) } \\
\hline & 1 & 2 & 3 & 4 & 5 & 6 & 7 & 8 & 9 & 10 & 11 & 12 \\
\hline 1. Edad & - &,- 255 &,$- 356^{* * *}$ &,$- 277^{* *}$ &,$- 394^{* * *}$ & 089 & ,082 &,- 159 &, 130 &,- 074 &,- 057 &,$- 184^{*}$ \\
\hline 2. Ayuda & & - & ,119 & , 125 &, 064 &,- 043 & ,107 & ,205 & ,098 & ,210 & 052 & ,072 \\
\hline 3. Salud Percibida & & & - &, $378^{* * *}$ & $448^{* * *}$ & $243^{* *}$ &, $181^{*}$ &, $199^{*}$ &, 147 &, $367^{* * *}$ &, $221^{* *}$ &, $326^{* * *}$ \\
\hline 4. Actividad Física & & & & - & $399^{* * *}$ & 161 & $221^{*}$ & 171 & ,141 &, $316^{* * *}$ & ,113 &, $307^{* * *}$ \\
\hline 5. Autoeficacia & & & & & - &, $287^{* *}$ &, $381^{* * *}$ & 164 & $259^{* *}$ &, $521^{* * *}$ &, $330^{* * *}$ &, $514^{* * *}$ \\
\hline 6. Autoestima & & & & & & - &, $574^{* * *}$ &, $221^{* *}$ &, $460^{* * *}$ &, $529^{* * *}$ &, $351^{* * *}$ &, $516^{* * *}$ \\
\hline 7. BP -Autoaceptación & & & & & & & - & $199^{*}$ &, $504^{* * *}$ &, $626^{* * *}$ &, $571^{* * *}$ &, $790^{* * *}$ \\
\hline 8. BP - Rel. Positivas & & & & & & & & - &, $188^{*}$ &, $353^{* * *}$ &, $302^{* * *}$ & $270^{* *}$ \\
\hline 9. BP - Autonomía & & & & & & & & & - &, $502^{* * *}$ &, $252^{* *}$ &, $379^{* * *}$ \\
\hline 10. BP - Dominio Medio & & & & & & & & & & . & $475^{* * *}$ &, $670^{* * *}$ \\
\hline 11. BP - Crec. Personal & & & & & & & & & & & - &, $582^{* * *}$ \\
\hline 12. BP- Propósito Vida & & & & & & & & & & & & - \\
\hline
\end{tabular}




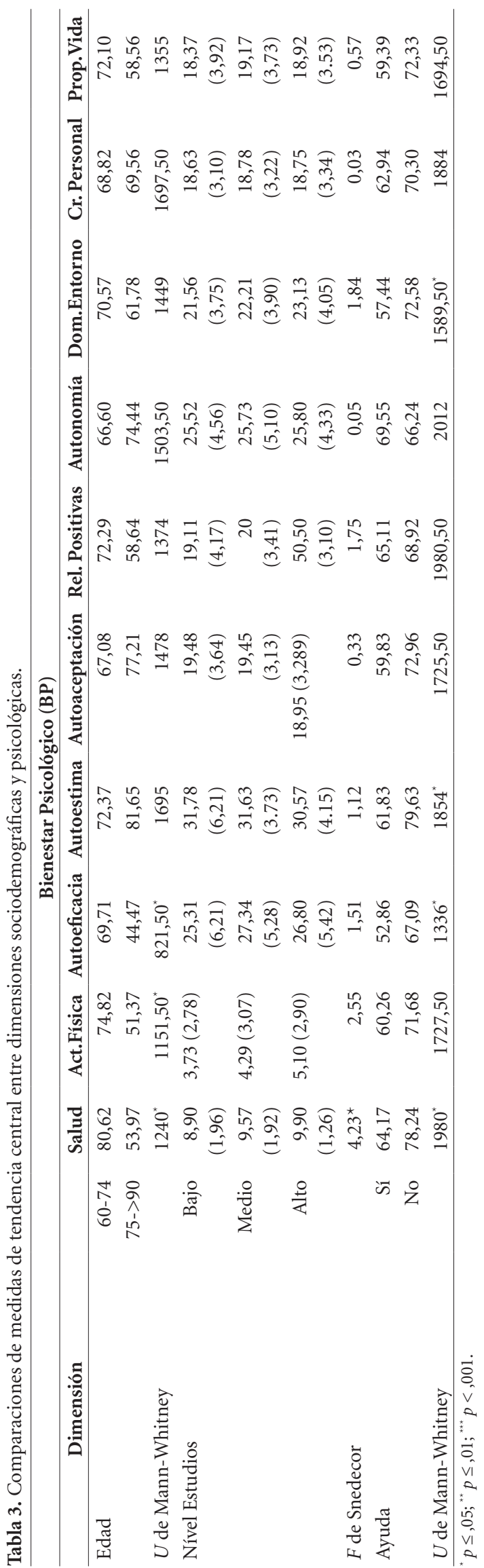

bles dependientes, considerándose dos bloques de posibles predictores. En el primer bloque, se decidió controlar la edad, la actividad física y la condición de tener ayuda para las AVD debido a la relación observada con el bienestar psicológico en los análisis preliminares. En el segundo bloque se introdujeron las variables psicológicas así como el nivel de salud percibida.

En un primer momento, al analizar los coeficientes de Durbin-Watson y colienalidad muestran la existencia de independencia de errores y no multicolinealidad entre las variables de los modelos.

Los resultados de los modelos de regresión jerárquica apuntan que todos los modelos incrementaron significativo de la varianza explicada al incluir las dimensiones psicológicas y de salud. Además de presentar un cambio en $R^{2}$, (puntuaciones entre 0,046 y 0,428 ), exceptuando el modelo de regresión de relaciones positivas, el cual no muestra diferencias significativas en el $R^{2}\left(R^{2}\right.$ corregida $=0,046, F(3,105)=1,87 p=0,093)$, por lo cual, explicaría el 4,6\% de la varianza del bienestar psicológico atendiendo a la influencia de los efectos principales. De este modo, los modelos con mayor grado de explicación del bienestar psicológico son aquellos centrados en dominio del medio, propósito de vida y autoaceptación.

La autoestima es la variable más influyente, y en segundo lugar la autoeficacia. Ambas explican el BP, atendiendo a las subescalas de autoaceptación, dominio del medio y propósito de vida $(t$ $=3,570, p=0,001),(t=4,827, p \leq 0,001)$. Por otro lado, la autoestima explica la autonomía $(t$ $=3,537, p \leq 0,001)$ y crecimiento personal $(t=$ $3,451, p=0,001)$. Asimismo, se observa que las personas que muestran mayores niveles de autoestima autoeficacia presentan mayores niveles de bienestar psicológico.

\section{Discusión}

Los objetivos del presente estudio eran caracterizar y analizar el perfil sociodemográfico de una muestra de personas mayores no dependientes, examinar las relaciones entre el bienestar psicológico y dimensiones sociodemográficas, de salud (actividad física y salud percibida) y psicológicas (autoestima y autoeficacia) y explorar los posibles factores que explican la vivencia de bienestar psicológico.

En primer lugar, se ha observado que la mayoría de las personas mayores de la muestra estaban casadas, residiendo mayoritariamente en su propia casa con su pareja, sin presentar algún 
Tabla 4. Coeficientes del análisis de regresión lineal múltiple con el bienestar como variable dependiente.

\begin{tabular}{|c|c|c|c|c|c|}
\hline & \multicolumn{5}{|c|}{ Bienestar psicológico: Autoaceptación } \\
\hline & $\mathbf{R}^{2}$ & B & $\beta$ & t & $\mathbf{P}$ \\
\hline Bloque 1. Variables de control & ,082 & & & & \\
\hline Edad & & ,058 & ,139 & 1,379 &, 171 \\
\hline Actividad Física & & ,261 & ,235 & 2,311 & ,023 \\
\hline Tiene ayuda & & $-1,001$ &,- 146 & $-1,509$ & , 134 \\
\hline Bloque 2. Predictores BP & $\Delta R^{2}, 324$ & & & & \\
\hline Autoestima & & ,389 & ,457 & 5,297 &, 000 \\
\hline Autoeficacia & &, 183 & ,327 & 3,196 & ,002 \\
\hline \multirow[t]{3}{*}{ Salud percibida } & &,- 134 &,- 072 & $-0,763$ & ,447 \\
\hline & \multicolumn{5}{|c|}{ Bienestar psicológico: Relaciones Positivas } \\
\hline & $\mathbf{R}^{2}$ & B & $\beta$ & $\mathbf{t}$ & $\mathbf{P}$ \\
\hline Bloque 1. Variables de control & ,050 & & & & \\
\hline Edad & &,- 044 &,- 090 & $-0,884$ & ,379 \\
\hline Actividad Física & & ,182 & ,141 & 1,362 & ,176 \\
\hline Tiene ayuda & &,- 594 &,- 074 & $-0,756$ & ,451 \\
\hline Bloque 2. Predictores BP & $\Delta R^{2}, 046$ & & & & \\
\hline Autoestima & & ,207 & ,208 & 1,962 & ,053 \\
\hline Autoeficacia & &,- 021 &,- 033 & $-0,261$ & ,794 \\
\hline \multirow[t]{3}{*}{ Salud percibida } & & ,269 & ,124 & 1,065 & ,289 \\
\hline & \multicolumn{5}{|c|}{ Bienestar psicológico: Autonomía } \\
\hline & $\mathbf{R}^{2}$ & B & $\beta$ & $\mathbf{t}$ & $\mathbf{P}$ \\
\hline Bloque 1. Variables de control & ,030 & & & & \\
\hline Edad & & ,089 & ,146 & 1,413 &, 160 \\
\hline Actividad Física & & ,244 & ,152 & 1,452 & , 149 \\
\hline Tiene ayuda & &,- 256 &,- 026 & $-0,259$ & ,796 \\
\hline Bloque 2. Predictores BP & $\Delta \mathrm{R}^{2}, 175$ & & & & \\
\hline Autoestima & & ,432 & ,349 & 3,537 &, 001 \\
\hline Autoeficacia & & , 184 & ,227 & 1,938 & ,055 \\
\hline \multirow[t]{3}{*}{ Salud percibida } & & ,070 & ,026 & 0,238 &, 812 \\
\hline & \multicolumn{5}{|c|}{ Bienestar psicológico: Dominio del Medio } \\
\hline & $\mathbf{R}^{2}$ & B & $\beta$ & $\mathbf{t}$ & $\mathbf{P}$ \\
\hline Bloque 1. Variables de control &, 134 & & & & \\
\hline Edad & &, 020 & 039 & 0,395 & ,694 \\
\hline Actividad Física & & ,380 & ,286 & 2,876 & ,005 \\
\hline Tiene ayuda & & $-1,563$ &,- 189 & $-2,002$ &, 048 \\
\hline Bloque 2. Predictores BP & $\Delta \mathrm{R}^{2}, 428$ & & & & \\
\hline Autoestima & & ,391 & ,384 & 4,632 &, 000 \\
\hline Autoeficacia & & ,244 & ,363 & 3,682 &, 000 \\
\hline Salud percibida & & ,203 & ,091 & 0,997 &, 321 \\
\hline
\end{tabular}

grado de dependencia o patología que lleven a necesitar ayuda para las AVD. Relativamente al nivel de estudios, tal como apuntan diversos estudios nacionales e internacionales ${ }^{19,23,24,30}$ poseer estudios superiores está asociado a mejores niveles de salud percibida debido a que estas personas muestran tener más recursos para lidiar con situaciones problemáticas a través de las estrategias adecuadas de afrontamiento y autorregulación, propiciando un mayor bienestar. Con respecto al nivel de actividad física y frecuencia de la misma, y de manera parecida a lo encontrado en muestras similares a las de este estudio ${ }^{30,31}$, la mayoría realizaban una actividad física ligera como andar, tonificación muscular o estiramientos evidenciando así efectos positivos en la salud percibida y en el bienestar.

En segundo lugar, se observó que las personas con mayor edad tienden a presentar una menor 
Tabla 4. Coeficientes del análisis de regresión lineal múltiple con el bienestar como variable dependiente.

\begin{tabular}{|c|c|c|c|c|c|}
\hline & \multicolumn{5}{|c|}{ Bienestar psicológico: Crecimiento Personal } \\
\hline & $\mathbf{R}^{2}$ & B & $\beta$ & $\mathbf{t}$ & $\mathbf{P}$ \\
\hline Bloque 1. Variables de control & ,031 & & & & \\
\hline Edad & &,- 012 &,- 027 & $-0,264$ & ,792 \\
\hline Actividad Física & & 147 & ,129 & 1,229 & ,222 \\
\hline Tiene ayuda & &,- 543 &,- 077 & $-0,773$ & ,441 \\
\hline Bloque 2. Predictores BP & $\Delta \mathrm{R}^{2}, 179$ & & & & \\
\hline Autoestima & & 299 & ,340 & 3,451 &, 001 \\
\hline Autoeficacia & & ,106 &, 184 & 1,570 & ,119 \\
\hline \multirow[t]{3}{*}{ Salud percibida } & & ,264 & ,137 & 1,274 & ,206 \\
\hline & \multicolumn{5}{|c|}{ Bienestar psicológico: Propósito de Vida } \\
\hline & $\mathbf{R}^{2}$ & B & $\beta$ & t & $\mathbf{P}$ \\
\hline Bloque 1. Variables de control & , 140 & & & & \\
\hline Edad & &,- 051 &,- 107 & $-1,101$ & ,273 \\
\hline Actividad Física & & ,333 & ,263 & 2,666 &, 009 \\
\hline Tiene ayuda & & $-1,047$ &,- 134 & $-1,427$ & ,157 \\
\hline Bloque 2. Predictores BP & $\Delta \mathrm{R}^{2}, 420$ & & & & \\
\hline Autoestima & & ,389 & ,399 & 4,827 &, 000 \\
\hline Autoeficacia & & ,224 &, 351 & 3,570 &, 001 \\
\hline Salud percibida & & 084 & 039 & 0,432 & ,666 \\
\hline
\end{tabular}

autoeficacia, resultados que discrepan con la literatura. Los estudios iniciales de Bandura ${ }^{8}$ y posteriores $^{18,42}$ revelan que la única dimensión de la autoeficacia que disminuye con la edad es la autoeficacia en la salud. Esta relación inversa entre la edad y la autoeficacia en la salud puede está relacionada con la propia percepción de salud. Así, y de acuerdo con el estudio de Wilson et al. ${ }^{43}$, las emociones positivas aumentan con la edad $y$, generalmente, las emociones negativas están asociadas a una mala salud. Estas emociones negativas y la disminución de la autoeficacia puede ser explicado por los estereotipos y autoestereotipos que, en ocasiones, son asumidos e influyen negativamente a la percepción de la persona mayor ${ }^{23,24,44}$. Por tanto, estos estereotipos y autoestereotipos influyen en la autoestima y autoeficacia, limitando y disminuyendo el esfuerzo e implicación en las actividades que realiza, resultando, una pérdida progresiva de interés y de las propias capacidades. Así, estas pérdidas favorecen el sentimiento de vulnerabilidad, originando, una situación de dependencia, y por ende, disminuyendo el bienestar ${ }^{44,45}$.

Por otro lado, se ha observado una relación positiva entre la salud percibida y el $\mathrm{BP}^{22}$, las personas que perciben una buena salud, experimentan sentimientos y emociones positivas favoreciendo niveles elevados de autoeficacia y autoestima, permitiendo a la persona poseer creencias sobre sus propias capacidades promoviendo conductas saludables y potenciando la situación de autonomía. En este sentido, tal y como apuntan Fernández-Ballesteros ${ }^{23}$ y Reigal y Videra ${ }^{26}$, la salud percibida está relacionada con la autoeficacia, produciendo de este modo, beneficios físicos y psicosociales, promoviendo la motivación, participación, adhesión y continuidad de los comportamientos saludables así como, la participación activa en actividades físicas, sociales y recreativas, y consecuentemente, la mejora del grado de autonomía y de bienestar ${ }^{21}$.

En tercer lugar, en este estudio se ha explorado algunos factores que pueden explicar el bienestar psicológico. A pesar de los cambios surgidos en el proceso de envejecimiento a nivel físico, cognitivo, emocional y social, se observa que la ligera disminución en el grado de bienestar observado, no está relacionada con la edad sino con otros factores ${ }^{46}$. En un estudio con mayores dominicanos de Molina y Meléndez ${ }^{15}$ revelaron que no existían diferencias en el BP según la edad. Por otro lado, en el estudio presente se observó una relación positiva entre las subescalas del BP y las variables psicológicas a estudiar, sugiriendo la autoestima y la autoeficacia como variables predictoras del bienestar psicológico. Estas variables son importantes para conseguir un buen reajuste emocional y adaptativo $^{8}$, tanto a nivel físico, psicológico y social en la edad adulta. Además, intervienen en la preven- 
ción y promoción de la salud $d^{18,21,23,24}$, así como, en la propia percepción de la misma y, en última instancia en la percepción del propio bienestar.

En conclusión, el bienestar psicológico está influenciado por los diferentes factores, sobre todo, por la autoeficacia y la autoestima, que a su vez, están relacionadas con otras variables como la salud percibida y la actividad física. De este modo, la participación en actividades físicas, recreativas y sociales promueven el bienestar, y en su última instancia el envejecimiento exitoso.

En cuanto a las limitaciones del estudio, cabe señalar el número de participantes de la muestra puede considerarse reducido, sin embargo los estudios realizados presentan muestras y resultados similares. Asimismo, para futuras investigaciones es de interés integrar una muestra mayor de personas mayores no dependientes y no participativas

\section{Colaboradores}

M Mesa-Fernández, J Pérez-Padilla, C Nunes y S Ménendez contribuyeron en la concepción, investigación, metodología y reacción final del manuscrito.

\section{Agradecimientos}

Este artigo es financiado por Fondos Nacionales de la Fundação para a Ciência e a Tecnologia (FCT) de Portugal en el ámbito del proyecto UID/SOC. de programas educativos, recreativos, culturales y/o de salud, aportando una visión más completa acerca de las personas mayores no dependientes integrando una muestra con un número mayor de participantes compuesta por personas "activas" y "no activas". De este modo, se podrían realizar diferencias entre grupos en las variables estudiadas según la participación en programas.

Aún con las limitaciones indicadas, este trabajo apoya la idea de que tanto la autoestima, como la autoeficacia y el bienestar psicológico deben ser dimensiones a considerar en el ámbito del estudio del envejecimiento con éxito. Ambos constructos han sido evaluados por instrumentos validados por lo que los resultados presentados pueden ofrecer claves relevantes para la intervención en esta población.

\section{Referencias}

1. Organización Mundial de la Salud. Envejecimiento activo: un marco político. Rev Esp Geriatr Gerontol 2002; 37(Supl. 2):74-105.

2. Organización Mundial de la Salud (OMS). Envejecimiento y ciclo de vida. [accesado 2015 Mayo 20]. Disponible en: http://www.who.int/ageing/about/facts/es/

3. European Commission \& Economic Policy Committee. The 2015 Ageing Report. Underlying Assumptions and Projection Methodologies. Brussels: European Commission Directorate-General for Economic and Financial Affairs; 2014.

4. Instituto Nacional de Estadística (INE). Proyecciones de la población de España 2014-2064. Madrid: INE; 2015.

5. del Castillo PC. 2012, Año Europeo del Envejecimiento Activo y la Solidaridad Intergeneracional. Humanismo $y$ trabajo social 2012; 11:101-117.

6. Baltes PB. Theoretical propositions of Life-span developmental psychology: On the dynamics between growth and decline. Dev Psychol 1987; 25(5):611-626.

7. Lima M. Envelhecimento e perdas: Como posso não me perder? Psychologica 2004; 35:133-145.

8. Bandura A. Self-efficacy: the exercise of control. New York: Freeman; 1997.

9. Ryff CD. Happiness is everything, or is it? Explorations on the meaning of psychological well-being. J Pers Soc Psychol 1989; 57(6):1069-1081.

10. Ryff CD. Psychological Well-Being in Adult Life. Curr Dir Psychol Sci 1995; 4(4):99-104.

11. Ryff CD. Optimizing well-being: the empirical encounter of two traditions. J Pers Soc Psychol 2002; 82(6):1007-1022.

12. Ryff CD, Magee WJ, Kling KC, Wing EH. Forging macro-micro linkages in the study of psychological well-being. In: Ryff CD, Marshall VW, editors. The self and society in aging processes. New York: Springer-Verlag; 199. p. 247-278. 
13. Meléndez JC, Tomás JM, Navarro E. Análisis del bienestar en la vejez según la edad. Rev Esp Geriatr Gerontol 2008; 43(2):90-95.

14. Meléndez JC, Tomás JM, Navarro E. Actividades de la vida diaria y bienestar y su relación con la edad y el género en la vejez. Anales de Psicología 2011; 27(1):164-169.

15. Molina C, Meléndez JC. Bienestar psicológico en envejecientes de la República Dominicana. Rev Iberoamer Gerontol Geriatr 2006; 22(3):97-105.

16. Tomás JM, Oliver, A, Navarro E, Meléndez JC, Molina C. Comparación del bienestar psicológico de personas mayores dominicanas y españolas. Rev Esp Geriatr Gerontol 2009; 44(4):180-185.

17. Mantovani E, Lucca S. Neri A. Autoavaliação negativa de saúde em idosos de cidades com diferentes níveis de bem-estar econômico: dados do Estudo FIBRA. Cien Saude Colet 2015; 20(12):3653-3668.

18. Fernández-Ballesteros R, Díez-Nicolás J, Caprara GV, Barbaranelli C, Bandura A. Determinants and structural relation of perceived personal efficacy to perceived collective efficacy. Appl Psychol 2002; 51(1):107-125.

19. Simões A, Ferreira JA, Lima MP, Pinheiro M, Vieira C, Matos A, Oliveira A. O bem-estar subjectivo dos idosos: Factores sócio-demográficos e de personalidade. In Simões, A. Oliveira A, Vieira C, Alcoforado L, Lima MP, Gaspar MF, editores. Modelos e práticas em educação de adultos. Coimbra: Núcleo de Assistência Psicológica e de Formação de Adultos; 2001. p. 301-320.

20. Simões A, Ferreira JA, Lima A, Pinheiro MR, Vieira C, Matos A, Oliveira A. O bem-estar subjetivo dos adultos: Um estudo transversal. Rev Port Pedagog 2003; 31(1):530.

21. Silva L, Farias L, Oliveira T, Rabelo D. Atitude de idosos em relação à velhice e bem-estar psicológico. Revi Kairós 2012; 15(3):119-140.

22. Vivaldi F, Barra E. Bienestar Psicológico, Apoyo Social Percibido y Percepción de Salud en Adultos Mayores. Ter. Psicol 2012; 30(2):23-29.

23. Fernández-Ballesteros R. Active Aging. Contributions from psychology. Toronto: Hogrefe \& Huber Publisher; 2008.

24. Caprara M. La promoción del envejecimiento activo. In: Fernández-Ballesteros R, editor. Psicología de la vejez. Una gerontología aplicada. Madrid: Pirámide; 2009. p. 337-361.

25. Navarro A, Bueno B, Buz J, Mayoral P. Percepción de autoeficacia en el afrontamiento de los problemas y su contribución en la satisfacción vital de las personas mayores. Rev Esp Geriatr Gerontol 2006; 41(4):222-227.

26. Reigal RE, Videra A. Frecuencia de práctica física y autoeficacia en la tercera edad. Rev Int Med Cienc Act Fís Deporte 2013; 13(49):107-120.

27. Queiroz B, Coqueiro R, Neto J, Borgatto A, Barbosa A, Fernandes M. Inatividade física em idosos não institucionalizados: estudo de base populacional. Cien Saude Colet 2014; 9(8):3489-3496.

28. McAuley E, Jerome G, Elavsky S, Marquez D, Ramsey S. Predicting long-term maintenance of physical activity in older adults. Prev Med 2003; 37(2):110-118.

29. Nishiguchi S, Yamada M, Tanigawa T, Sekiyama K, Kawagoe T, Suzki M, Yoshikawa S, Abe N, Otsuka Y, Nakai R, Aoyama T, Tsuboyama T. A 12-week physical and cognitive exercise program can improve cognitive function and neutral efficiency in community-dwelling older adults: A randomized controlled trial. J Am Geriatr Soc 2015; 63(7):1355-1363.
30. Pérez S. Calidad de vida en personas mayores. Madrid: Dykinson; 2006.

31. García AJ, Marín M, Bohórquez MR. Autoestima como variable psicosocial predictora de la actividad física en personas mayores. Rev Psicol Dep 2012; 21(1):195-200.

32. Wallace J, Buchner D, Grohaus L, Leville S, Tyl, L, LaCroix A, Wagner E. Implementation and effectiveness of community-based health promotion program for older adults. J Gerontol A Bio Sci Med Sci 1998; 53(4):M301-306.

33. Cohen G, Perlstein S, Chapline J, Kelly J, Firth K, Simmens S. The impact of professionally conducted cultural programs on the physical health, mental health, and social functioning of older adults. Gerontologist 2006; 46(6):726-734.

34. Lutosa L, Oliveira L, Santos L, Guedes R, Parentoni A, Pereira L. Efeito de um programa de treinamento funcional no equilíbrio postural de idosas da comunidade. Fisioter Pesqui 2010; 17(2):153-156.

35. Pérez-Albéniz A, Pascual A, Navarro M, Lucas-Molina, B. Más allá del conocimiento. El impacto de un programa educativo universitario para mayores. Aula $a b-$ ierta 2015; 43(1):54-60.

36. Irigay T, Schneider R. Participação de Idosas em uma universidade da terceira idade: Motivos e mudanças. Psic Teor Pesq 2008; 24(2):211-216.

37. Requejo Osorio A, Rumbo B, Cid X. Programas universitários para idosos. In: Requejo Osório A, Cabral Pinto F, editores. As pessoas idosas. Contexto social e intervenção educativa. Lisboa: Instituto Piaget; 2007. p. 307-321.

38. Clemente A, Tartaglini M, Stefani D. Apoio social, bienestar psicológico y salud percibida en personas de edad. Perspec psicol 2008; 5(2):47-53.

39. Fernández-Ballesteros R, Reig A, Zamarrón MD. Evaluación en psicogerontología. In: Fernández-Ballesteros R, editor. Psicología de la vejez. Una Psicogerontología aplicada. Madrid: Pirámide; 2009. p. 35-96.

40. Rosenberg M. Society and the adolescent self-image. Princeton: Princeton University Press; 1965.

41. Díaz D, Rodríguez C, Van Dierendonck D. Adaptación española de las Escalas de Bienestar Psicológico de Ryff. Psicothema 2006; 18(3):572-577.

42. Lachman M, Agrigoroaei S. Promoting functional health in midlife and old age: Long-term protective effects of control beliefs, social support, and physical exercise. PLoS One 2010; 5(10):1-9.

43. Wilson RS, Krueger KR, Gul L, Bienias JL, Mendes de Leon CF, Evans DA. Neuroticism, extraversion, and mortality in a defined population of older persons. Psychosom Med 2005; 67(6):841-845.

44. Sáez N, Meléndez, J, Aleixandre M. Los estereotipos en los ancianos: Un estudio empírico y sus resultados. Rev Psicol Edu 1994; 14:75-89.

45. Santos A. Controle Percebido: um estudo comparativo entre homens e mulheres. Rev Bra Ter Cogn 2005; 1(1):1-10.

46. Fernández-Ballesteros R. Geropsychology - An Applied Field for the "1st Century". Eur Psychol 2006; 11(4):312-323.

Artigo apresentado em 27/12/2016

Aprovado em 26/04/2017

Versão final apresentada em 28/04/2017 